\title{
Liraglutide With or Without Metformin Ameliorates Liver Function and Fatty Liver in Obese Patients with Type 2 Diabetes Mellitus
}

Tadashi Iwao*, Kenji Sakai and Eiji Ando

Department of Medicine, Iwao Hospital, Hita, Japan

*Corresponding author: Tadasi Iwao, M.D, Department of Medicine, Iwao Hospital, 2-4-29 Tansou, Hita 877-0012, Japan, Tel: +81-973-22-6161; Fax: +81-973-22-6258; E-mail: iwao@oregano.ocn.ne.jp

Rec date: Aug 05, 2015, Acc date: Aug 26, 2015, Pub date: Aug 28, 2015

Copyright: @ 2015 Iwao T, et al. This is an open-access article distributed under the terms of the Creative Commons Attribution License, which permits unrestricted use, distribution, and reproduction in any medium, provided the original author and source are credited.

\begin{abstract}
Objective: To evaluate efficacy of 24 weeks administration of liraglutide with or without metformin on liver parameters, visceral adipose tissue (VAT) area and liver-to-spleen attenuation (L/S) ratio between non-obese and obese patients with type 2 diabetes mellitus.
\end{abstract}

Methods: A total of 57 patients with type 2 diabetes: non-obese group $(\mathrm{N}=30)$ and obese group $(\mathrm{N}=27)$ were enrolled. HbA1c, body weight, aspartate aminotransferase (AST), alanine aminotransferase (ALT), and VAT area and $\mathrm{L} / \mathrm{S}$ ratio were measured before and after the treatment.

Results: In the two groups, sustained and significant reductions were observed in $\mathrm{HbA} 1 \mathrm{c}$, and body weight. A similar reduction was observed in AST and ALT level, but the change was greater in obese group than in non-obese group ( $p<0.05, p<0.05$, respectively). Although reduction in VAT area did not differ in both groups, the change of L/S ratio was greater in obese subjects than in non-obese subjects $(p<0.05)$. In all patients, multiple regression analysis showed that the reduction in ALT was significantly correlated with the reduction in VAT area $(95 \%$ confidential interval $0.15-0.69 ; p<0.01$ ), whereas either the changes in $\mathrm{HbA} 1 \mathrm{c}$, body weight and L/S ratio or the presence of dyslipidemia were not significant.

Conclusion: Liraglutide with or without metformin ameliorates liver function and reduces fat tissue on the liver particularly in obese patients with type 2 diabetes mellitus. The potential mechanism might be due to decreases VAT area by liraglutide with or without metformin.

Keywords: Liraglutide; Liver function; Type 2 diabetes mellitus; Visceral adipose tissue

\section{Abbreviations:}

AST: Aspartate Aminotransferase; ALT: Alanine Aminotransferase; BMI: Body Mass Index; CT: Computed Tomography; L/S: Liver-toSpleen Attenuation; VAT: Visceral Adipose Tissue

\section{Introduction}

Steatosis and steatohepatitis is highly prevalent in patients with type 2 diabetes mellitus, particularly in obese subjects [1]. According to recent studies, the majority of uncomplicated steatosis is nonprogressive; approximately $20 \%-30 \%$ of patients will develop nonalcoholic steatohepatitis, a more aggressive necro-inflammatory phenotype associated with increased risk for advanced fibrosis predisposing to cirrhosis, portal hypertension, decompensated liver failure and hepatocellular carcinoma [2-4]. It is reported that steatohepatitis is common liver disease, because of the rising prevalence of obesity [5].

Glucagon-like peptide-1 (GLP-1) is an incretin hormone. The glucose-lowering actions of GLP-1 are glucose dependent, which limits the risk of hypoglycemia [6,7]. GLP-1 induces insulin secretion and reduces glucagon secretion, resulting in a potent blood-glucose lowering action. In addition, GLP-1 slows gastric emptying and induces satiety [8], leading to decreased energy intake and weight loss $[9,10]$. Liraglutide is a once-daily human GLP-1 analogue with a high degree (97\%) of amino-acid-sequence identity with native human GLP-1 [11]. Several multinational trials have shown that liraglutide is well tolerated, improves glycemic control with a low risk of hypoglycemia associated with weight loss $[12,13]$.

Limited information is currently available effect of liraglutide on liver parameters. A recent study has shown that liragulutide as monotherapy or added to metformin reduces fat mass in visceral adipose tissue(VAT) area associated with weight loss as compared glimepiride in type 2 diabetes mellitus [14]. In 2012, Ohki et al. retrospectively reported that liraglutide improves liver function associated with reduction in body weight among nonalcoholic fatty liver disease with type 2 diabetes mellitus [15]. The pathogenesis of non-alcoholic hepatitis is considered by two-hit theory $[16,17]$. In the first hit, over-nutrition and lack of exercise induces VAT accumulation, resulting in increased secretion of free fatty acid, decreased adiponectin and increased tumor necrosis factor- $\alpha$. These results cause fatty change of the liver. In the second step (two hit), oxidative stress provokes hepatocyte injury.

We therefore hypothesized that beneficial process with on liraglutide with and without metformin on liver function and steatosis is more pronounced in obese patients with type 2 diabetes. If so, several factors, such as glycemic control, weight loss, reduction in VAT 
area, increment of liver-to-spleen attenuation (L/S) ratio, and the presence of dyslipidemia may correlate with reduction of aspartate aminotransferase (AST) and/or alanine aminotransferase (ALT). The present study was carried out to clarify the process.

\section{Materials and Methods}

\section{Patients and Liraglutide dosing}

Between September 1, 2010 and August 31, 2012, a total of consecutive 72 patients with type 2 diabetes who received liraglutide were included. 10 patients were naïve, 43 patients were switched from insulin therapy and 19 patients were switched from sulfonylurea therapy. There were 39 non-obese patients less than body mass index $(B M I)<25$ and 33 obese patients $(B M I \geq 25)$. Exclusion criteria were stage 4 or 5 nephropathy, viral hepatitis, pregnancy, malignancy and age more than 80 years old.

The patients receiving liraglutide (Novo Nordisk, Bagsvaerd, Denmark) underwent a 1 week dose escalation, starting at $0.3 \mathrm{mg}$ once daily with weekly increments of $0.3 \mathrm{mg}$, reaching a final daily dose of $0.9 \mathrm{mg}$ by the end of the third week under approved Japanese dose. If an adequate glycemic control was achieved, increments of dose of liraglutide were limited.

We intended to treat to achieve target HbAlc level less than 7.0\% after 24 weeks administration of liraglutide. To achieve this target, we added metformin, if necessary.

This study was conducted in accordance with local institutional review board approval and conducted in accordance with the Declaration of Helsinki. All patients received full explanations of the use of liraglutide in written informed concept.

\section{Measurements}

HbAlc was measured, using high-performance liquid chromatography according to an assay certified by the Japan Diabetes Society, at baseline and at 4,8,12,16, 20 and 24 weeks after starting liraglutide therapy. In this manuscript, we converted them to National Glycohemoglobin Standardization Program equivalent value (\%) calculated by the formula HbAlc (\%) = HbAlc (Japan Diabetes Society, \%) $+0.4 \%$ [18]. Similarly, AST, ALT, body weight and body mass index (BMI) were also measured. AST and ALT were measured, using ultraviolet absorption spectrophotometry. Safety assessments included hypoglycemic episodes based on symptoms and selfmonitoring of blood sugar (blood glucose less than $70 \mathrm{mg} / \mathrm{dL}$ ).

VAT area and L/S ratio were measured by computed tomography (CT) scans (Toshiba, Alexion 0.4, Japan) before and after 24 weeks liraglutide with and without metformin therapy. VAT area was determined using intraperitoneal CT scans at the position of the navel level, using software (Fat view, version 1.1.2, Daito, Co. Ltd, Aichi, Japan). About L/S ratio, in an arbitrary section, we measured the mean of the CT values more than $2 / 3$ of each liver and spleen size, then, $\mathrm{L} / \mathrm{S}$ ratio was defined the division of the value as the liver $\mathrm{CT}$ value/spleen $\mathrm{CT}$ value. These values were measured by radiological technician who had no clinical information of the patients. The diagnosis of fatty liver was made if $\mathrm{L} / \mathrm{S}$ ratio less than 1.10 [19].

\section{Intra-assay of coefficient of variation of the parameters}

Intra-assay of coefficient of variation of HbA1c, AST, ALT, VAT area and $\mathrm{L} / \mathrm{S}$ ratio, was $0.5 \%, 0.1 \%, 0.3 \%, 0.03 \%$ and $1.5 \%$ for 20 samples, respectively.

\section{Statistical analyses}

The data were expressed as mean \pm standard deviation. For univariate analyses, we used t-test for continuous variables and chi square test and Fisher's exact test for the proportion of discrete variables between obese patients and mon-obese patients with type 2 diabetes.

In each group, the values of body weight, $\mathrm{HbAlc}$, AST and ALT at 4, $8,12,16,20$ and 24 weeks after administration of liraglutide with or without metformin were compared it with baseline using paired t-test. Similarly, the values of VAT area and L/S ratio after administration of 24 weeks liraglutide with and without metformin were compared it with baseline using paired t-test. We also compared the changes in body weight, HbAlc, AST, ALT, VAT area and L/S ratio between the two groups by t-test.

In all patients, multiple regression analysis used to assess the relationship between the presence of dyslipidemia, the changes in HbA1c, body weight, VAT area, L/S ratio and the changes in AST and ALT after administration of 24 weeks liraglutide with or without metformin. All p-values were two-tailed, and p-values $<0.05$ were considered statistically significant. Statistical analyses were carried out using Excel 2012 statistical software package (version 1.15, Tokyo, Japan version 1.10, Tokyo, Japan, Social Survey Research Information Co., Ltd).

\section{Results}

\section{Patients and clinical characteristics}

In non-obese group, a total of nine patients were further excluded, because missing data was in six patients and drop out was in three patients due to gastrointestinal symptom, rejection of continued liraglutide therapy, and acute cholangitis. Consequently, a total of 30 patients (77\%) were enrolled.

\begin{tabular}{|l|l|l|l|}
\hline & BMI $<\mathbf{2 5}(\mathbf{n}=\mathbf{3 0})$ & BMI $\mathbf{2 5}(\mathbf{n}=\mathbf{2 7})$ & $p$ value \\
\hline Age (year) & $66.2 \pm 12.2$ & $55.4 \pm 16.2$ & 0.001 \\
\hline Gender (M/F) & $17 / 13$ & $15 / 14$ & 0.461 \\
\hline Duration (Year) & $8.6 \pm 5.8$ & $8.8 \pm 9.2$ & 0.899 \\
\hline Prior to treatment & & & \\
\hline
\end{tabular}


Citation: Iwao T, Sakai K, Ando E (2015) Liraglutide With or Without Metformin Ameliorates Liver Function and Fatty Liver in Obese Patients with Type 2 Diabetes Mellitus. J Diabetes Metab 6: 598. doi:10.4172/2155-6156.1000598

Page 3 of 6

\begin{tabular}{|l|l|l|l|}
\hline (Naïve/insulin/sulfonylurea) & $5 / 20 / 5$ & $7 / 17 / 3$ & 0.819 \\
\hline Height $(\mathrm{cm})$ & $158 \pm 10$ & $161 \pm 8$ & 0.18 \\
\hline Body weight (kg) & $54.4 \pm 9.0$ & $75.5 \pm 10.8$ & 0 \\
\hline Body mass index & $21.6 \pm 2.1$ & $28.9 \pm 2.6$ & 0 \\
\hline HbA1c (\%) & $7.2 \pm 1.3$ & $7.8 \pm 1.5$ & 0.135 \\
\hline Rate of dyslipidemia & $9(30.0 \%)$ & $17(63.0 \%)$ & 0 \\
\hline AST (U/L) & $25 \pm 6$ & $36 \pm 28$ & 0.033 \\
\hline ALT (U/L) & $24 \pm 15$ & $42 \pm 36$ & 0.017 \\
\hline VAT area (cm2) & $92 \pm 49$ & $148 \pm 69$ & 0.001 \\
\hline L/S ratio & $1.15 \pm 0.11$ & $0.97 \pm 0.21$ & 0 \\
\hline Rate of fatty liver & $12(40.0 \%)$ & $20(74.1 \%)$ & 0 \\
\hline
\end{tabular}

Table 1: Clinical characteristic between non-obese patients and obese patients with type 2 diabetes

In obese group, a total of six patients were excluded, missing data was in five patients and transfer to another clinic in one patient. Eventually, a total of 27 patients (81\%) were enrolled.

There was no significant difference to missing patients between the two groups $(\mathrm{p}=0.233)$.

As shown in Table 1, there were no significant differences in gender, duration of the disease, prior to treatment before liraglutide administration, height and HbAlc. In contrast, body weight, BMI, AST, ALT and VAT area was significantly higher in obese group than in non-obese group $(\mathrm{p}<0.01, \mathrm{p}<0.01, \mathrm{p}<0.05, \mathrm{p}<0.05$ and $\mathrm{p}<0.01$, respectively). Furthermore, the rate of the presence of dyslipidemia and fatty liver was significantly higher in obese group than in nonobese group ( $\mathrm{p}<0.01$ and $\mathrm{p}<0.01$, respectively) while $\mathrm{L} / \mathrm{S}$ ratio was significantly lower in obese group than in non-obese group $(\mathrm{p}<0.01)$.

\section{Dose of liraglutide, metformin, and changes in parameters}

We intended to treat to achieve targeting HbAlc level less than $7.0 \%$ after 24 weeks administration of liraglutide with or without metformin. Although reduction in HbAlc was bit higher in obese group than in non-obese group, the change did not reach the level of statistical significance. $(\mathrm{p}=0.635)$ in the two groups, the dose of liraglutide $(\mathrm{mg} / \mathrm{kg})$ and dose of metformin $(\mathrm{mg} / \mathrm{kg})$ was equivalent (Table 2$)$.

In Table 2, the changes in parameters after 24 weeks administration of liraglutide with or without metformin between non-obese subjects and obese patients are summarized. At the end point, although reduction in $\mathrm{HbAlc}$, body weight, BMI, and VAT area was similar in the two groups, reduction in AST and ALT was significantly greater in obese group than in non-obese group $(\mathrm{p}<0.05$ and $\mathrm{p}<0.05$, respectively). The increase in $\mathrm{L} / \mathrm{S}$ ratio is significantly higher in the obese group than in non-obese group $(\mathrm{p}<0.05)$.

As demonstrated in Figure 1, sustained and significant reduction in both $\mathrm{HbAlc}$ and body weight was observed during liraglutide with or without metformin. The difference in $\mathrm{HbAlc}$ was not significant at each point in the two groups, but the reduction in body weight was significantly higher in obese group the in non-obese group. As illustrated in Figure 2, sustained and significant reduction in both AST and ALT was observed during liraglutide with or without metformin in obese group, while the reduction in AST and ALT is transient in nonobese group.

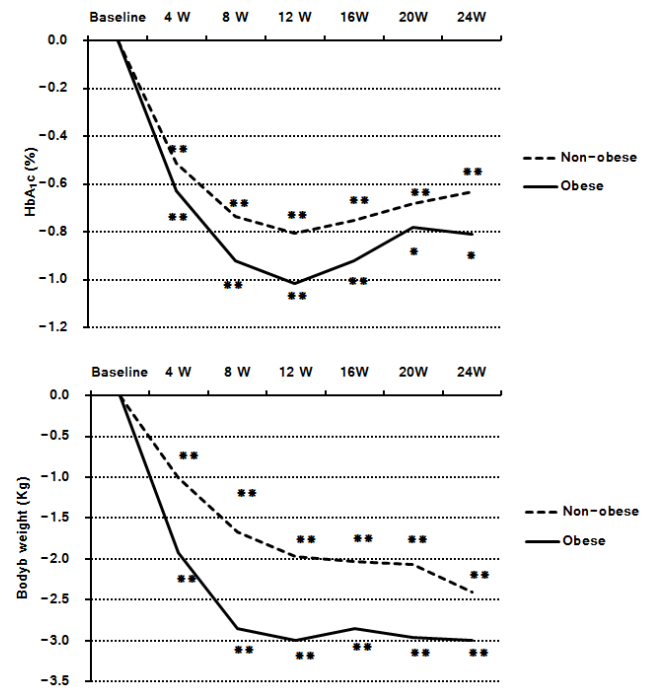

Figure 1: Effect of 24 weeks liraglutide or added metformin therapy in $\mathrm{HbAlc}$ level between obese and non-obese patients with type 2 diabetes (upper panel) in body weigh level between obese and nonobese patients with type 2 diabetes (lower panel). ${ }^{\star} p<0.05$ from baseline, ${ }^{* *} \mathrm{p}<0.01$ from baseline 
Page 4 of 6

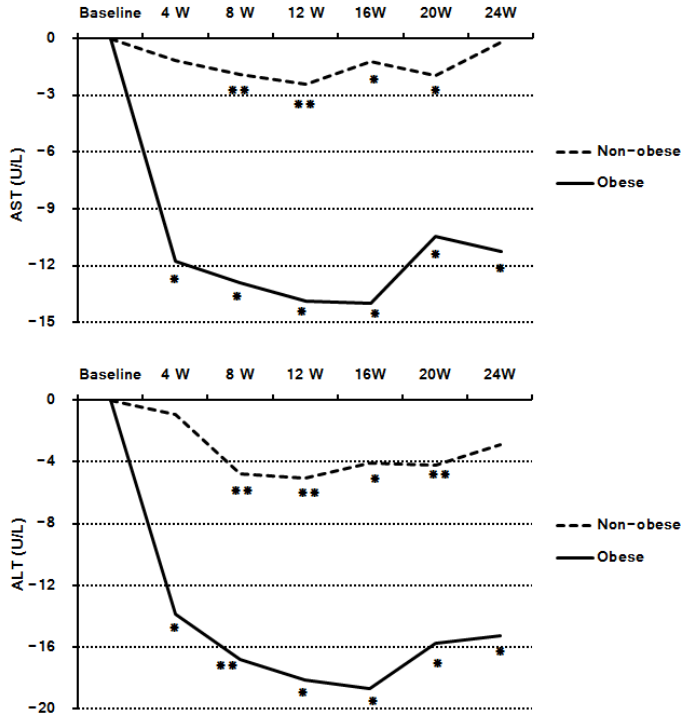

Figure 2: Effect of 24 weeks liraglutide or added metformin therapy in AST level between obese and non-obese patients with type 2 diabetes (upper panel) in ALT level between obese and non-obese patients with type 2 diabetes (lower panel). ${ }^{\star} \mathrm{p}<0.05$ from baseline, ${ }^{* *} \mathrm{p}<0.01$ from baseline

\begin{tabular}{|c|c|c|c|}
\hline & $\begin{array}{l}\text { BMI }<25 \\
(\mathrm{n}=30)\end{array}$ & $\begin{array}{l}\text { BMI } \geq 25 \\
(n=27)\end{array}$ & p value \\
\hline $\begin{array}{l}\text { Dose } \\
\text { liraglutide } \\
(\mathrm{mg} / \mathrm{kg})\end{array}$ & $0.012 \pm 0.005$ & $0.010 \pm 0.03$ & 0.146 \\
\hline $\begin{array}{l}\text { Dose } \\
\text { metformin of } \\
(\mathrm{mg} / \mathrm{kg})\end{array}$ & $8.29 \pm 13.08$ & $7.23 \pm 9.49$ & 0.729 \\
\hline HbA1c (\%) & $\begin{array}{l}6.6 \pm 0.9(-0.6 \pm 1.2 \\
\%)^{\star \star}\end{array}$ & $7.0 \pm 1.1(-0.8 \pm 1.5 \%)^{*}$ & 0.635 \\
\hline $\begin{array}{l}\text { Body weight } \\
\text { (kg) }\end{array}$ & $\begin{array}{l}52.0 \pm 0.9(-2.4 \pm 3.1 \\
\%)^{* *}\end{array}$ & $\begin{array}{l}72.5 \pm 12.6(-3.0 \pm 5.0 \\
\%)^{* *}\end{array}$ & 0.586 \\
\hline $\begin{array}{l}\text { Body mass } \\
\text { index }\end{array}$ & $\begin{array}{l}20.7 \pm 0.5(-1.0 \pm 1.3 \\
\%)^{* *}\end{array}$ & $\begin{array}{l}27.7 \pm 3.4(-1.2 \pm 1.9 \\
\%)^{* \star}\end{array}$ & 0.588 \\
\hline AST (U/L) & $\begin{array}{l}24.6 \pm 11.2(-0.2 \pm 8.8 \\
\%)\end{array}$ & $\begin{array}{l}24.9 \pm 11.0(-11.3 \pm 27.4 \\
\%)^{*}\end{array}$ & 0.041 \\
\hline ALT (U/L) & $\begin{array}{l}20.8 \pm 11.9(-2.9 \pm 9.4 \\
\%)\end{array}$ & $\begin{array}{l}26.4 \pm 11.0(-15.3 \pm 32.0 \\
\%)^{*}\end{array}$ & 0.048 \\
\hline VAT area $(\mathrm{cm} 2)$ & $\begin{array}{l}79.8 \pm 47.4 \quad(-12.2 \pm \\
17.7 \%)^{\star *}\end{array}$ & $\begin{array}{l}130.0 \pm 56.6 \quad(-18.4 \pm \\
32.1 \%)^{\star \star}\end{array}$ & 0.364 \\
\hline $\mathrm{L} / \mathrm{S}$ ratio & $\begin{array}{l}1.11 \pm 0.11 \quad(-0.04 \pm \\
0.13 \%)\end{array}$ & $\begin{array}{l}1.05 \pm 0.16(0.08 \pm 0.23 \\
\%)^{*}\end{array}$ & 0.019 \\
\hline
\end{tabular}

*: $p<0.05$ from the baseline, ${ }^{* *}: p<0.01$ from the baseline. The figures in parentheses are the difference from the baseline

Table 2: Dose of liraglutide, dose of metformin, and the changes in parameters after 24 weeks administration of liraglutide with or without metformin between non-obese subjects and obese patients
VAT area was significantly reduced in both non-obese and obese group after 24 weeks administration of liraglutide with or without metformin $(p<0.01$ and $p<0.01$, respectively, Table 2$)$. No significant change was observed in L/S ratio in non-obese group after 24 weeks administration of liraglutide with or without metformin. In contrast, a significant increment in L/S ratio was observed in obese group $(\mathrm{p}<0.05$, Table 2).

As shown in Table 1, 20 patients $(74 \%)$ had fatty liver in obese group, i.e., L/S ratio less than 1.1. After 24 weeks administration of liraglutide with or without metformin, only 14 patients (52\%) had fatty liver $(\mathrm{p}<0.05)$

Multiple regression analysis of the relationship between the changes in $\mathrm{HbAlc}$, body weight, VAT area and L/S ratio, the presence of dyslipidemia and the change in liver parameters

As shown in Table 3, when all patients were included, there was a significant correlation was observed between the change in VAT area and the change in ALT (95\% confidential interval 0.15-0.69; $\mathrm{p}<0.01$ ) whereas the changes in $\mathrm{HbAlc}$, body weight, $\mathrm{L} / \mathrm{S}$ ratio and the rate of dyslipidemia did not correlate.

\begin{tabular}{|l|l|l|}
\hline & $\mathbf{9 5 \%}$ confidential interval & p value \\
\hline HbA1c $(\%)$ & $-2.25-7.82$ & 0.273 \\
\hline Body weight $(\mathrm{kg})$ & $-1.52-1.68$ & 0.919 \\
\hline VAT area (cm2) & $0.15-0.69$ & 0.003 \\
\hline L/S ratio & $-16.9-56.7$ & 0.283 \\
\hline Rate of dyslipidemia & $-17.1-6.49$ & 0.37 \\
\hline
\end{tabular}

Table 3: Multiple regression analysis of the relationship between the changes in $\mathrm{HbAlc}$, body weight, VAT area, L/S ratio, and the presence of dyslipidemia and the change in ALT after 24 weeks administration of liraglutide with or without metformin $(\mathrm{N}=57)$

Similar trend was observed in AST level, but the difference did not just reach to the level of statistical significance ( $95 \%$ confidential interval -0.01-0.48; $\mathrm{p}=0.057$ ).

\section{Safety assessment and adverse effect}

In the follow-up period, major hypoglycemia and minor hypoglycemia did not occur in all patients. As mentioned above, only one patient was not able to continue liraglutide therapy due to liraglutide-induced gastrointestinal symptom.

\section{Discussion}

In this study, we were able to demonstrate that 24 weeks administration of liraglutide with or without metformin improved liver function in particular in obese group compared with non-obese group. This was supported by current observation in which 24 weeks administration of liraglutide with or without metformin a sustained reduction in AST and ALT level in obese group. This finding partially supports of the previous report [15].

In our patients, similar reduction in $\mathrm{HbAlc}$ level between obese group and non-obese group was observed, the impact of reduction in AST and ALT level was greater in the former. This is because the baseline value of AST and ALT level was significantly higher in obese 
patients than in non-obese patients with type 2 diabetes mellitus. In general, obese patients have frequently liver injury, such as nonalcoholic steatohepatitis and non-alcoholic fatty liver disease [20]. This is further supported by the current observation in which the rate of the presence of dyslipidemia and fatty liver was significantly higher in obese group than in non-obese group.

We confirmed that liraglutide with or without metformin reduced VAT areas associated with body weight in non-obese group and obese group [14]. This finding is an important advantage of a long term liraglutide with or without metformin therapy, because insulin therapy [21] or other anti-hyperglycemic therapies, such as pioglitazone do not generally have such effects on body composition [22,23]. Total body weight and total body fat have been shown to increase with the pioglitazone [22,23]. For example, increases in body weight and total body fat of 3.9 and $3.5 \mathrm{~kg}$ respectively, were observed over 24 weeks with pioglitazone treatment [22]. However, the amount of VAT area did not change [22,23].

Jendle et al. have shown that liraglutide or added metformin increased the L/S ratio increased (0.10) from baseline, while the unchanged ratio in the glimepiride group. In this study, in accordance with that study [14], we observed liraglutide with or without metformin increased the L/S ratio increased $(0.09)$ from baseline in obese group. In these patients, fatty liver was observed in $74 \%$. These previous [14] and current findings suggest that liraglutide with or without metformin have possibly reduced hepatic steatosis.

Our important finding in this study is that liraglutide with or without metformin induced-beneficial effect on liver function was due to the reduction in VAT area (first hit). This finding was supported by the current observation in which multiple regression analysis showed that there was a significant correlation between the reduction in VAT area and the reduction in ALT (second hit). Although the reduction in VAT area and the reduction in AST did not just reach to the level of statistical significance. The reduction in AST and ALT was not correlated with the reduction in $\mathrm{HbAlc}$, the reduction body weight, increment in $\mathrm{L} / \mathrm{S}$ ratio and the presence of dyslipidemia. It is not clear in this study whether such a beneficial effect on liver function is due to liraglutide alone or synergic effect liraglutide and metformin. Nevertheless, liraglutide with or without metformin therapy are promising particularly in obese patients with type 2 diabetes mellitus not only glycemic control but also improvement of fatty liver.

We were able to demonstrate that liraglutide with or without metformin therapy reduces hepatic steatosis. This is supported by the current observation in which the change in the rate of fatty liver was significantly decreased in obese group after liraglutide with or without metformin therapy. Indeed, there was a substantial evidence that optimal cut off value of $\mathrm{L} / \mathrm{S}$ ratio is 1.1 to exclude liver steatosis (area under the ROC curve $=0.886$ ) [19].

In this study, the dose of liraglutide and /or metformin was similar in the two groups. Nonetheless, glycemic control was relatively worse in obese group than in non-obese group. This means that, as we previously reported [24], the strategy of glycemic control is different from obese patients to non-obese patients with type 2 diabetes mellitus.

Concerning to adverse effect, we experienced that only one patient had liraglutide-induced gastrointestinal symptoms, resulting in discontinued the therapy (1.4\%). In overall, liraglutide with or without metformin therapy was well tolerated. During the study, hypoglycemia was not occurred in the two groups.
In conclusion, a long term liraglutide with or without metformin therapy in patients with type 2 diabetes mellitus improved liver function associated with the reduction in hepatic steatosis in particular in obese patients with type 2 diabetes. This beneficial effect on the liver would be potentially explained by reduction in VAT area. Further large studies are needed to address whether liraglutide-based therapy is superior to other antidiabetic-based therapy on liver-related event or mortality.

\section{Acknowledgement}

Authors declare that there is no financial or non-financial conflict of interest and competing interests.

\section{References}

1. Bhatt HB, Smith RJ (2015) Fatty liver disease in diabetes mellitus. Hepatobiliary Surg Nutr 4: 101-108.

2. Serfaty L, Lemoine M (2008) Definition and natural history of metabolic steatosis: clinical aspects of NAFLD, NASH and cirrhosis. Diabetes Metab 34: 634-637.

3. Basaranoglu M, Basaranoglu G, Sentürk H (2013) From fatty liver to fibrosis: a tale of "second hit" World J Gastroenterol 19: 1158-1165.

4. Singh S, Allen AM, Wang Z, Prokop LJ, Murad MH, et al. (2015) Fibrosis progression in nonalcoholic fatty liver vs. nonalcoholic steatohepatitis: a systematic review and meta-analysis of paired-biopsy studies. Clin Gastroenterol Hepatol 13: 643-654.

5. Clark JM, Brancati FL, Diehl AM (2003) The prevalence and etiology of elevated aminotransferase levels in the United States. Am J Gastroenterol 98: 960-967.

6. Chia CW, Egan JM (2008) Incretin-based therapies in type 2 diabetes mellitus. J Clin Endocrinol Metab 93: 3703-3716.

7. Drucker DJ, Nauck MA (2006) The incretin system: glucagon-like peptide-1 receptoragonists and dipeptidyl peptidase- 4 inhibitors in type 2 diabetes. Lancet 368: 1696-1705.

8. Flint A, Raben A, Astrup A, Holst JJ (1998) Glucagon-like peptide 1 promotes satiety and suppresses energy intake in humans. Journal of Clinical Investigation 101: 515-520.

9. Van Cauter E, Mestrez F, Sturis J, Polonsky KS (1992) Estimation of insulin secretion rates from C-peptide levels: comparison of individual and standard kinetic parameters for C-peptide clearance. Diabetes 41: 368-377.

10. Zander M, Madsbad S, Madsen JL, Holst JJ (2002) Effect of 6-week course of glucagon like peptide 1 on glycaemic control, insulin sensitivity, and beta-cell function in type 2 diabetes: a parallel-group study. Lancet 359: 824-830.

11. Agersø H, Jensen LB, Elbrønd B, Rolan P, Zdravkovic M (2002) The pharmacokinetics, pharmacodynamics, safety and tolerability of NN2211, a new long-acting GLP-1 derivative, in healthy men. Diabetologia 45: 195-202.

12. Garber A, Henry R, Ratner R, Garcia-Hernandez PA, Rodriguez-Pattzi H, et al. (2009) Liraglutide versus glimepiride monotherpy for type 2 diabetes (LEAD-3 Mono): a randomised, 52-week, phase III, doubleblind, parallel-treatment trial. Lancet 373: 473-481.

13. Nauck M, Frid A, Hermansen K, Shah NS, Tankova T, et al. (2009) Efficacy and safety comparison of liraglutide, glimepiride, and placebo, all in combination with metformin in type 2 diabetes. Diabetes Care 32: 84-90.

14. Jendle J, Nauck MA, Matthews DR, Frid A, Hermansen K, et al. (2009) Weight loss with liraglutide, a once-daily human glucagon-like peptide-1 analogue for type 2 diabetes treatment as monotherapy or added to metformin, is primarily as a result of a reduction in fat tissue. Diabetes Obes Metab 11: 1163-1172.

15. Ohki T, Isogawa $A$, Iwamoto $M$, Ohsugi $M$, Yoshida $H$, et al. (2012) The effectiveness of liraglutide in nonalcoholic fatty liver disease patients with 
Citation: Iwao T, Sakai K, Ando E (2015) Liraglutide With or Without Metformin Ameliorates Liver Function and Fatty Liver in Obese Patients with Type 2 Diabetes Mellitus. J Diabetes Metab 6: 598. doi:10.4172/2155-6156.1000598

Page 6 of 6

type 2 diabetes mellitus compared to sitagliptin and pioglitazone. Scientific World Journal 2012: 496-453.

16. Day CP, James OF (1988) Steatohepatitis: a tale of two "hits"? Gastroenterology 114: 842-845.

17. Mehta K, Van Thiel DH, Shah N, Mobarhan S (2002) Nonalcoholic fatty liver disease: pathogenesis and the role of antioxidants. Nutr Rev 60: 289-293.

18. Kashiwagi A, Kasuga M, Araki E, Oka Y, Hanafusa T, et al. (2013) International clinical harmonization of glycated hemoglobin in Japan: from Japan Diabetes Society to National Glycohemoglobin Standardization Program values. J Diabetes Invest 3: 39-40.

19. Kan H, Kimura Y, Hyogo H, Fukuhara T, Fujino H, et al. (2014) Noninvasive assessment of liver steatosis in non-alcoholic fatty liver disease. Hepatol Res 44: E420-E427.

20. Bedogni G, Miglioli L, Masutti F, Tiribelli C, Marchesini G, et al. (2005) Prevalence of and risk factors for nonalcoholic fatty liver disease: the Dionysos nutrition and liver study. Hepatology 42: 44-52.
21. Russell-Jones D, Khan, R (2007) Insulin-associated weight gain in diabetes-causes, effects and coping strategies. Diabetes Obes Metab 9: 799-812.

22. Smith SR, deJonge L, Volaufova J, Li Y, Xie H, et al. (2005) Effect of pioglitazone on body composition and energy expenditure: a randomized controlled trial. Metab Clin Exp 54: 24-32.

23. Basu A, Jensen MD, McCann F, Mukhopadhyay D, Joyner MJ, et al. (2006) Effects of pioglitazone versus glipizide on body fat distribution, body water content, and hemodynamics in type 2 diabetes. Diabetes Care 29: 510-514.

24. Iwao T, Sakai K, Ando E (2014) Relative contribution of insulin secretion and sensitivity at different stages of glucose tolerance: non-obese versus obese Japanese subjects. Intern Med 53: 383-390. 